\title{
Breakdown of the standard Perturbation Theory and Moving Boundary Approximation for "Pulled" Fronts
}

\author{
Ute Ebert $^{1}$ and Wim van Saarloos ${ }^{2}$ \\ ${ }^{1}$ CWI, Postbus 94079, 1090 GB Amsterdam, The Netherlands \\ ${ }^{2}$ Instituut-Lorentz, Universiteit Leiden, Postbus 9506, 2300 RA Leiden, \\ The Netherlands
}

November 5, 2018

\begin{abstract}
The derivation of a Moving Boundary Approximation or of the response of a coherent structure like a front, vortex or pulse to external forces and noise, is generally valid under two conditions: the existence of a separation of time scales of the dynamics on the inner and outer scale and the existence and convergence of solvability type integrals. We point out that these conditions are not satisfied for pulled fronts propagating into an unstable state: their relaxation on the inner scale is power law like and in conjunction with this, solvability integrals diverge. The physical origin of this is traced to the fact that the important dynamics of pulled fronts occurs in the leading edge of the front rather than in the nonlinear internal front region itself. As recent work on the relaxation and stochastic behavior of pulled fronts suggests, when such fronts are coupled to other fields or to noise, the dynamical behavior is often qualitatively different from the standard case in which fronts between two (meta)stable states or pushed fronts propagating into an unstable state are considered.
\end{abstract}




\section{Introduction}

For a pattern in two or more dimensions that naturally can be divided into domains and "domain walls" separating them, a much used analytical approach is a moving boundary or effective interface approximation [1, 2, 3, 4, 5, 6, 6, 8. This seems appropriate, when the width of the domain wall, front, interface, or transition zone is much smaller than the typical length scale of the pattern and when the dynamics of the pattern on long space and time scales occurs through the motion of these interfaces. The moving boundary approximation amounts to treating these fronts or transition zones as a mathematically sharp interface or boundary. In other words, their width is taken to be zero and their internal degrees of freedom are eliminated. We shall henceforth use the word boundary or interface to denote this zero width limit and use the word front when we look at a scale where its internal structure can be resolved.

Moving boundary approximations (MBA's) are ubiquitous in the theory of pattern formation: they arise in most analytical approaches to late stage coarsening [9, 10], in the analysis of interface dynamics in dendritic growth and viscous fingering [11, 12, 13, 14, 15, 16, 17, step dynamics at surfaces [18, 19, 20], thermal plumes [21, 22], in chemical wave dynamics [23], combustion fronts [3], etc.

The main physical idea underlying the derivation of a MBA is that the front itself can on large length and time scales be viewed as a well-defined coherent structure which can be characterized by its coordinates and a few effective parameters, such as its velocity or a mobility coefficient. This idea plays a role for many coherent structures, like vortices, or pulse-type solutions like sources, sinks, solitons, etcetera [24, 25, 26, 27, 28]. The response of a coherent structure to an external driving force or noise [29, 30] or the interaction between them can frequently be derived by a perturbative expansion about the isolated coherent structure solution. Often the effective parameters (a diffusion coefficient, a mobility or an effective interaction force) can be derived from a solvability condition. A solvability condition expresses that a linear equation of the form $L \phi_{1}=g_{1}$, where the linear operator $L$ results from linearizing about the isolated coherent structure solution, is solvable provided $g_{1}$ is orthogonal to the kernel (null space) of $L$. In other words, the requirement for such an equation to be solvable is that that $g_{1}$ is orthogonal to the left zero mode $\chi$ of $L$.

Although this is hardly ever mentioned explicitly, there are two important 
implicit assumptions underlying such approximations, namely $(a)$ that there is a separation of time scales between the motion of the front as a whole and its internal dynamics, and $(b)$ that the internal dynamics of the front is determined by the nonlinear front region itself, so that the solvability type integrals are dominated by the contributions from this finite region, and hence donot diverge.

The issue that we address in this paper is that while the above conditions are satisfied for the familiar MBA for bistable fronts and also for so-called pushed fronts, they are not for so-called pulled fronts. We will indeed discuss several related properties of pulled fronts which bear on this: $(i)$ the divergence of the solvability integrals, with the concommittant breakdown of a MBA or of the derivation of the response functions of the front, like a diffusion or mobility coefficient; $(i i)$ the shift of the dynamically dominant zone from the interior to the leading edge of the front, that causes the solvability integrals to diverge; (iii) the fact that the stability spectrum of planar pulled front solutions is gapless; $(i v)$ the recently discovered universal slow power law relaxation of planar pulled fronts [31]. We will initially focus our discussion on the derivation of a MBA, but as we shall see our observations and conclusions apply equally well to essentially any perturbative analysis of a pulled front.

The crucial feature of the standard moving boundary problem is that the boundary conditions are local in space and time - e.g., the growth velocity of an interface is a function of the instantaneous local temperature and curvature of the interface. Usually, some of the boundary conditions are associated with conservation laws, like the conservation of heat, and so they can often be guessed from physical considerations.

If there is a separation of spatial scales, then such a MBA applies only if there is also a separation of time scales between the internal dynamics of the front and the dynamics of the outer bulk fields. E.g., if the internal front modes relax on a time scale $\tau$, and one considers a front of width $W$, propagation velocity $v$ and typical curvature $\kappa$, then a MBA becomes appropriate in the regime $\kappa W \ll 1, v \kappa \tau \ll 1$. Such a well-defined relaxation time $\tau$ of a front on the inner scale actually exists only if the relaxation is exponential in time. In this case, $\tau$ is the inverse of the gap in the spectrum of the stability modes of the planar front. Just like multiple scale and amplitude expansions [32, 33, 34] are based on projecting all rapidly decaying gapped modes onto the slow one (the center manifold), the MBA or effective interface approximation can be thought of as projecting a problem with fronts onto the slow interfacial dynamics. 
However, if the stability spectrum of the planar front is gapless, the internal modes of the front relax algebraically in time. Thus there is no characteristic time $\tau$ for the internal modes, no separation of time scales and no standard MBA, no matter how thin the front is. The internal dynamics of such a pulled front is actually slaved to the evolution of its leading edge on the outer scale, which motivates the term "pulling". Note that despite its different temporal behavior, it is not at all visible on an instantaneous picture of a front, whether it is bistable, pushed or pulled.

In a problem where the starting equations are partial differential equations, the derivation of the MBA can often be done analytically using by now standard methods. One should keep in mind, however, that MBA's can be equally powerful in situations where the approximation can not be derived cleanly by starting from a partial differential equation and applying standard methods. E.g., in crystal growth the interfacial boundary conditions are determined on a molecular scale, where for a rough interface the molecular processes are so fast that after some coarse graining, we can describe the interface for many purposes as a sharp interface whose response to changes in temperature and concentration are instantaneous [35]. Similar considerations apply to coarsening interfaces or combustion fronts.

In the next section, we will first summarize the necessary essentials of the stability and relaxation properties of pulled fronts. Then, in section 3 we illustrate the issue by following the standard derivation of a MBA for the type of coupled equations that have in recent years been used in a phasefield type formulation of solidification problems. In section 4 we then discuss the conditions under which such a type of analysis applies in more detail, to identify the difficulties that arise when the front dynamics on the inner scale is changed from the usual bistable or pushed case to pulled. We then in section 5 generalize our findings to equations with higher derivatives and to coupled equations, that create uniformly translating fronts. We show that the usual route of deriving solvability conditions does work in general for bistable and pushed fronts, but not for pulled fronts. 


\section{Pulled fronts: Properties and statement of the problem}

When one considers a linearly unstable state, even a small perturbation about this unstable state grows out and spreads. We will confine our analysis to fronts emerging from a localized initial perturbation of the unstable state. One can calculate the asymptotic linear spreading velocity $v^{*}$ of such a perturbation simply from the linear dispersion relation $\omega(k)$ of the unstable modes according to [36, 37]

$$
\left.\frac{\mathrm{d} \omega(k)}{\mathrm{d} k}\right|_{k^{*}}=v^{*}, \quad \frac{\operatorname{Im} \omega\left(k^{*}\right)}{\operatorname{Im} k^{*}}=v^{*} .
$$

We furthermore will confine ourselves in this paper to fronts which asymptotically are uniformly translating. For these, $\omega^{*}$ and $k^{*}$ are purely imaginary, and we use the notation $k^{*}=i \lambda^{*}$. If the above equation admits more than one solution, the one corresponding to the largest value of $v^{*}$ is the relevant one. We refer for the derivation of these results to our recent paper [31], which we will quote as paper I below. Pulled fronts are those for which the asymptotic spreading velocity $v_{a s}$ of the nonlinear front equals this linear spreading velocity $v^{*}: v_{a s}=v^{*}$ [38, 39, 40, 11, 31]. A number of model equations for which fronts are pulled are discussed in paper I, but they also arise in the analysis of more complicated situations like pearling [42], the Couette-Taylor instability [43], Rayleigh-Bénard convection [44], the instabilities of wakes of bluff bodies, leading e.g. to von Karman instabilities [45], the emergence of global modes [46], liquid crystals [47], streamer discharge patterns [48], the competion of domains in the Kupers-Lortz instabilility [49], the emergence of domains near structural phase transitions [50], polymer patterns [51], superconducting fronts [52], error propagation [53], deposition models [54, step propagation [18], chaotic fronts in the complex Ginzburg-Landau equation [55, 56, 57], renormalization group analysis of disorder models [58], and the analysis of the Lyapunov exponents in kinetic models [59].

Fronts which propagate into an unstable state always are pulled if all the nonlinearities suppress the growth. If not all of them do, the asymptotic fronts speed $v_{a s}$ may become larger than $v^{*}: v_{a s}=v^{\dagger}>v^{*}$. The relaxation of such "pushed" fronts [38, 41, 31] is exponential with a characteristic relaxation time

$\tau$, that is finite [40, 31]. As we will discuss, for these the same perturbative schemes apply as for the familiar bistable fronts, and likewise for these a 
standard type MBA can be derived.

In paper I, we have shown that when a pulled front grows out of sufficiently steep initial conditions (decaying into the unstable state at least as $e^{-\lambda x}$ for $x \rightarrow \infty$ with some $\lambda>\lambda^{*}$ ), then the velocity of a front obeys a universal power law relaxation given by

$$
\begin{aligned}
v(t) & \equiv v^{*}+\dot{X}(t) \\
\dot{X}(t) & =-\frac{3}{2 \lambda^{*} t}+\frac{3 \sqrt{\pi}}{2 \lambda^{* 2} \sqrt{D} t^{3 / 2}}+O\left(\frac{1}{t^{2}}\right),
\end{aligned}
$$

where

$$
D=\left.\frac{i \mathrm{~d}^{2} \omega(k)}{2 \mathrm{~d} k^{2}}\right|_{k^{*}}
$$

is real and positive for uniformly translating front solutions. For the front profile, a similar power law relaxation holds, and the extension of these results to one-dimensional pattern forming fronts is given in [57]. The analysis reveals, that the power law relaxation emerges from the dynamics of the foremost part of the front where the dynamics is governed by the equations linearized about the unstable state. The dynamics in the nonlinear region is essentially slaved to this so-called leading edge. The very slow $1 / t$ power law relaxation of pulled fronts without characteristic time scale obviously implies that the separation of time scales, which is necessary for a MBA to be applicable, is missing.

While from this perspective it is already intuively obvious that a standard perturbation theory or MBA does not apply to pulled fronts, the arguments underlying the separation of time scales are hardly ever discussed explicitly in the literature on the derivation of a MBA. The purpose of this article therefore is to point out where the standard derivation breaks down and how this emerges at a more formal level. In such an approach, one generally encounters solvability type integrals of the form

$$
\int_{-\infty}^{\infty} d \xi e^{v \xi}\left(\frac{\partial \Phi_{0}}{\partial \xi}\right)^{2}
$$

or generalizations thereof - see e.g. (22), (27) or (37). Here $\xi=x-v t$ is a frame moving with the front with speed $v$, and $\Phi_{0}(\xi)$ is the associated planar front solution. The translation mode $\partial_{\xi} \Phi_{0}$ is a right zero mode of the linear operator $L$ emerging from linearization about the asymptotic front $\Phi_{0}$, and 
$e^{v \xi} \partial_{\xi} \Phi_{0}$ is a left zero mode of this operator. As we shall see, such solvability type integrals are well-defined and finite for bistable and pushed fronts, but diverge for pulled fronts, since the integrand does not converge for $\xi \rightarrow \infty$. In a way, the solvability integral still correctly distributes its weight over the dynamically important region, but for a pulled front, this region becomes semi-infinite, and therefore the integral diverges. Our discussion also shows why introduction of an ad-hoc cutoff in these integrals - an approach that has sometimes been considered in the literature - does not necessarily cure the problem.

\section{The derivation of a MBA from a phase field model}

In this section, we first follow the standard derivation of a moving boundary approximation (MBA) from a phase field model to highlight the assumptions and approximations along the way. We then analyze why and how the approximation breaks down for pulled fronts.

As an example, we study the "phase field model"

$$
\begin{aligned}
\partial u / \partial t= & \alpha \nabla^{2} u+\partial \phi / \partial t, \\
\varepsilon \partial \phi / \partial t= & \varepsilon^{2} \nabla^{2} \phi+f(\phi, u), \\
\text { where } \quad & f(\phi, u)=\phi(1-\phi)(\mu-\lambda u+\phi), \quad \lambda>0 .
\end{aligned}
$$

In the limit of zero front width $\epsilon \rightarrow 0$, this model for appropriate parameters $\alpha, \mu$ and $\lambda$ reduces to a moving boundary approximation for a solidification front, where we can think of $\phi$ as the order parameter field, while $u$ plays the role of the temperature. $\phi$ then varies from the stationary "liquid-like" solution $\phi \approx 0$ in one domain to another "solid-like" solution $\phi \approx 1$ in the other domain. Note that in contrast to [1], $\partial \phi / \partial t$ in (7) has a coefficient $\varepsilon$, not $\varepsilon^{2}$. This allows the front to have a velocity of order unity, so the velocity is nonvanishing already in the lowest order perturbation theory $O\left(\varepsilon^{0}\right)$. The $\partial \phi / \partial t$ on the r.h.s. in (6) models the generation of latent heat in the interfacial zone where $\phi$ changes rapidly.

Other choices for $f(\phi, u)$ can be found in the literature [2, 4, 61, 8] but the form (8) is most convenient for our present purpose. $f$ can be considered as 
the derivative of a "free energy" $F$,

$$
\begin{aligned}
& f(\phi, u)=-\frac{\partial F(\phi, u)}{\partial \phi}, \\
& F(\phi, u)=-\frac{(\mu-\lambda u) \phi^{2}}{2}-\frac{(1-\mu+\lambda u) \phi^{3}}{3}+\frac{\phi^{4}}{4} .
\end{aligned}
$$

Since $u$ varies on spatial and temporal scales of order unity, let us treat it as a constant for a moment on the small length scale $\varepsilon$ on which $\phi$ varies, and let us define $\bar{\mu}=\mu-\lambda u$. The connection with the phase field models for solidification is closest in the range $-1<\bar{\mu}<0$, when the function $F$ (which is like a Ginzburg-Landau free energy density), has two minima at $\phi=0$ and at $\phi=1$. When $\bar{\mu}=-1 / 2$, then $F(0, u)=F(1, u)=0$ and the two "phases" $\phi=0$ and $\phi=1$ are in equilibrium. So if we choose the bare parameter $\mu=-1 / 2$, then $u=0$ corresponds to the melting temperature, where (7) admits stationary front solutions with velocity $v=0$. For $\mu=-1 / 2$ but $u$ nonzero, the minima of $f$ shift relative to each other, and the order parameter front (7) moves. When $u$ is positive, the liquid like minimum at $\phi=0$ is the absolute minimum of $F$, and for $u$ negative the solid like minimum at $\phi=1$ is the absolute one. The front then will move such that the state with the lowest free energy extends. For $\bar{\mu}>0$ the state $\phi=0$ is linearly unstable; so we then deal with fronts propagating into unstable states which are pushed for $0<\bar{\mu}<1 / 2$ and pulled for $\bar{\mu}>1 / 2$ [39, 40, 31]. Though the interpretation of the model as a solidification model might be lost, we will illustrate the derivation of a MBA as a function of $\bar{\mu}$ for this example, and we will find that the method breaks down at the transition from pushed to pulled fronts at $\bar{\mu}=1 / 2$.

Let us now trace the steps of the approximation in more detail. The field $u$ (6) varies on a spatial scale of order unity, and the field $\phi$ (7) on a spatial scale of order $\varepsilon \ll 1$. A moving boundary approximation consists of first matching an inner expansion of the problem on scale $\varepsilon$ to an outer problem on scale 1 , and then letting $\varepsilon \rightarrow 0$ such that an effective moving boundary problem on the outer scale results. In the limit of $\varepsilon \rightarrow 0$, the interface might have a nonvanishing velocity and curvature on the outer length scale, so we allow for $v=O\left(\varepsilon^{0}\right)$ and $\kappa=O\left(\varepsilon^{0}\right)[62$.

Let us for simplicity consider the problem in two spatial dimensions $(x, y)$. On the outer scale, the fields are expanded in powers of $\varepsilon$ as

$$
u(x, y, t)=u_{0}(x, y, t)+\varepsilon u_{1}(x, y, t)+\ldots,
$$




$$
\phi(x, y, t)=\phi_{0}(x, y, t)+\varepsilon \phi_{1}(x, y, t)+\ldots .
$$

For a further analysis of these equations on the outer scale and their matching to the inner scale, we refer to the literature. Here we focus on the analysis of the $\phi$-front on the inner scale. First a coordinate system moving with the front is introduced, where $s$ measures the arc length of the interface in the tangential direction, and $\xi$ the direction in which $\phi$ varies and propagates. We put, e.g., $\xi=0$ at the place where $\phi=1 / 2$. The coordinate $\xi$ in the direction normal to the front is scaled with a factor $\varepsilon$, since the front width will be of order $\varepsilon$ in the limit $\varepsilon \rightarrow 0$. However, the coordinate $s$ is not scaled: along the front, the variation is assumed to be simple on length scales of the order of unity. For the inner expansion of the fields, one then writes

$$
\begin{aligned}
u(x, y, t) & =U_{0}(\xi, s, t)+\varepsilon U_{1}(\xi, s, t)+\ldots, \\
\phi(x, y, t) & =\Phi_{0}(\xi, s, t)+\varepsilon \Phi_{1}(\xi, s, t)+\ldots .
\end{aligned}
$$

The choice of coordinates can be illustrated when we consider a weakly curved front which locally propagates with a velocity $v(s, t)$ in the $x$ direction, so that

$$
s=y \quad, \quad \xi=\frac{x-X(s, t)}{\varepsilon} \quad, \quad X(s, t)=x_{0}+\int^{t} \mathrm{~d} t^{\prime} v\left(s, t^{\prime}\right) .
$$

In general, the front is curved and has a velocity $v$ and curvature $\kappa$ which varies locally but on the outer time scale $t$ and spatial scale $s$. They are therefore are expanded as

$$
\begin{aligned}
& v(s, t)=v_{0}(s, t)+\varepsilon v_{1}(s, t)+\ldots, \\
& \kappa(s, t)=\kappa_{0}(s, t)+\varepsilon \kappa_{1}(s, t)+\ldots .
\end{aligned}
$$

The differential operators in (7) then in the interior coordinates $(\xi, s)$ have the $\varepsilon$ expansion

$$
\begin{aligned}
\left.\varepsilon \frac{\partial}{\partial t}\right|_{(x, y)} & =\left.\varepsilon \frac{\partial}{\partial t}\right|_{(\xi, s)}-\left[v_{0}+\varepsilon v_{1}+\ldots\right] \frac{\partial}{\partial \xi}+O\left(\varepsilon^{2}\right) \\
\varepsilon^{2} \nabla^{2} & =\frac{\partial^{2}}{\partial \xi^{2}}+\varepsilon \kappa_{0} \frac{\partial}{\partial \xi}+O\left(\varepsilon^{2}\right) .
\end{aligned}
$$

Inserting the expanded operators into (7) and ordering in powers of $\varepsilon$ yields in order $\varepsilon^{0}$

$$
\frac{\partial^{2}}{\partial \xi^{2}} \Phi_{0}+v_{0} \frac{\partial}{\partial \xi} \Phi_{0}+f\left(\Phi_{0}, U_{0}\right)=0
$$


where $U_{0}$ is essentially constant on the inner scale $\xi$. In order $\varepsilon^{1}$ one finds

$$
L \Phi_{1}=-\left(\kappa_{0}+v_{1}\right) \frac{\partial}{\partial \xi} \Phi_{0}+\frac{\partial}{\partial t} \Phi_{0}-\left.\frac{\partial f\left(\Phi_{0}, U\right)}{\partial U}\right|_{U_{0}} U_{1}
$$

with the linear operator

$$
L \equiv\left(\frac{\partial^{2}}{\partial \xi^{2}}+v \frac{\partial}{\partial \xi}+\left.\frac{\partial f\left(\Phi, U_{0}\right)}{\partial \Phi}\right|_{\Phi_{0}}\right)
$$

Note that since $\Phi_{0}$ is a solution of the ordinary differential equation (19), its time dependence occurs solely through the variation the $U$ field.

Equation (20) is an inhomogeneous linear differential equation for the unknown field $\Phi_{1}$. If one has a left zero mode $\chi(\xi)$ of $L$ such that $\chi L=0=L^{\dagger} \chi$, where $L^{\dagger}$ is the adjoint operator defined through partial integrations, then (20) can be evaluated with a so-called "solvability" analysis by projection onto $\chi$ :

$$
\left(\kappa_{0}+v_{1}\right) \int_{-\infty}^{\infty} d \xi \chi \frac{\partial \Phi_{0}}{\partial \xi}+\left.\int_{-\infty}^{\infty} d \xi \chi \frac{\partial f\left(\Phi_{0}, U\right)}{\partial U}\right|_{U_{0}} U_{1}=\int_{-\infty}^{\infty} d \xi \chi \frac{\partial \Phi_{0}}{\partial t}
$$

There are clearly two important conditions for the identification of (22) with the common solvability condition: If the scalar products with $\chi$ exist, and if the temporal derivative $\partial_{t} \Phi_{0}$ of the zero order solution (19) can be neglected, then (22) expresses the first order velocity correction $v_{1}$ as a function of the local curvature $\kappa_{0}$, of the outer temperature field $\partial_{U} f U_{1}$ and of the zero order solution $\Phi_{0}$ (19). It is exactly at these two points that the analysis breaks down for pulled fronts. The violation of these conditions always happens concomitantly, as they are physically related.

Let us construct the left zero mode $\chi$ explicitly: It is well known, that the right zero mode of $L$ is the mode of infinitesimal translation $\partial_{\xi} \Phi_{0}: L \partial_{\xi} \Phi_{0}=0$. Since $L$ is nonhermitian, the left zero mode of $L$ is a right zero mode of the adjoint $L^{\dagger}$ of $L$,

$$
L^{\dagger} \chi(\xi)=0, \quad L^{\dagger} \equiv\left(\frac{\partial^{2}}{\partial \xi^{2}}-v \frac{\partial}{\partial \xi}+\left.\frac{\partial f\left(\Phi, U_{0}\right)}{\partial \Phi}\right|_{\Phi_{0}}\right)
$$

and $\chi \neq \partial_{\xi} \Phi_{0}(\xi)$. However, the left zero mode $\chi$ can be obtained by noting that the transformation

$$
\phi=e^{-v \xi / 2} \tilde{\phi}, \quad L \phi=\tilde{L} \tilde{\phi},
$$


with

$$
\tilde{L}=e^{v \xi / 2} L e^{-v \xi / 2}=\left(\frac{\partial^{2}}{\partial \xi^{2}}+\left.\frac{\partial f\left(\Phi, U_{0}\right)}{\partial \Phi}\right|_{\Phi_{0}}-\frac{v^{2}}{4}\right) .
$$

turns the problem into a hermitian eigenvalue problem. As a result the left zero eigenmode $\tilde{\chi}$ of $\tilde{L}$ is equal to the right zero eigenmode $e^{v \xi / 2} \partial_{\xi} \Phi_{0}$ of $\tilde{L}$. Transforming back to $L$, this yields for the left zero mode

$$
\chi=e^{v \xi} \partial_{\xi} \Phi_{0},
$$

as can also be verified by substitution. If we may ignore the term associated with the time derivative $\partial_{t} \Phi_{0}$ and insert the expression for $\chi$ into (22) we find

$$
v_{1}=-\kappa_{0}-\frac{\left.\int_{-\infty}^{\infty} d \xi e^{v \xi} \frac{\partial \Phi_{0}}{\partial \xi} \frac{\partial f\left(\Phi_{0}, U\right)}{\partial U}\right|_{U_{0}} U_{1}}{\int_{-\infty}^{\infty} d \xi e^{v \xi}\left(\frac{\partial \Phi_{0}}{\partial \xi}\right)^{2}} .
$$

If we furthermore ignore the term due to the coupling to the $u$ field, the expression $v_{1}=-\kappa_{0}$ is the familiar result of motion by mean curvature first derived within the context of continuum models by Allen and Cahn [60, 10].

The structure of the solvability analysis is generic for the perturbative expansion about a uniformly translating front. Although we have only considered the simplest type of model, and although refinements are possible [2], Eq. (27) captures the basic structure of the expression that one obtains in lowest order in a MBA: the relations between the velocity, curvature and temperature field $u$ of the front, which play the role of boundary conditions for the outer fields at the boundary in the zero width limit $\varepsilon \rightarrow 0$, contain solvability integrals of the form $\int d \xi e^{v \xi}\left(\partial_{\xi} \Phi_{0}\right)^{2}$. (Note that $\partial_{U} f$ in (27) contains a factor $\Phi_{0}$, that for $\xi \rightarrow \infty$ decays essentially like $\partial_{\xi} \Phi_{0}$.) Solvability integrals of this type essentially arise in any type of perturbative calculation, since they just express the solvability condition of the linear perturbation problem $L \Phi_{1}(\xi)=g_{1}(\xi)$ : the inhomogeneous term $g_{1}(\xi)$ has to be orthogonal to the left zero mode $\chi$ of the linear operator $L$.

\section{Violation of the two conditions underlying the MBA for pulled fronts}

We now discuss the conditions under which the MBA can be derived along the lines sketched above in more detail. 
Consider first the condition concerning the separation of time scales. For fronts between two linearly stable states (with $-1<\bar{\mu}<0$ in $f$ ), there is such a separation between the inner dynamics of the front and its displacement: In this case, the stability spectrum of planar front modes has a gap [31, 35], and all internal eigenmodes decay as $e^{-\omega_{n} t / \varepsilon}$ with eigenvalues $\omega_{n} \geq \omega_{1}=1 / \tau=O(1)$. Thus, in the limit $\varepsilon \rightarrow 0$, there is a clear separation of inner and outer time scales, and the adiabatic approximation (27) is justified on the outer time scale of order unity. Moreover, as discussed in paper I, for pushed fronts propagating into an unstable state the stability spectrum is also gapped, and therefore the separation of timescales necessary for the MBA to apply, does hold. However, the stability spectrum of pulled fronts is gapless, and as Eq. (2) of section II illustrates, pulled fronts show indeed a power law convergence to their asymptotic speed $v^{*}$. Clearly, then, the standard derivation of a MBA does not apply to pulled fronts.

The same conclusion also emerges from the properties of the solvability integrals themselves. For increasing $v$, the exponential factor $e^{v \xi}$ enhances the value of the integrand for large positive $\xi$, while suppressing the integrand for large negative $\xi$. We therefore now turn to fronts propagating into an unstable state for $\mu>0$ (for simplicity of notation, we use $u=0$ ), and investigate the behavior of the integrand for $\xi \rightarrow \infty$. The large $\xi$ asymptotics of $\Phi_{0}(\xi)$ follows directly from the o.d.e. (19) by noting that $f^{\prime}\left(\Phi_{0}(\infty)\right)=f^{\prime}(0)=\mu$, so that [31]

$$
\Phi_{0}(\xi) \stackrel{\xi \gg 1}{\simeq} \begin{cases}A_{1}(v) e^{-\lambda_{-} \xi}+A_{2}(v) e^{-\lambda_{+} \xi}, & v>v^{*}=2 \sqrt{\mu}, \\ (\alpha \xi+\beta) e^{-\lambda^{*} \xi}, & v=v^{*}=2 \sqrt{\mu}\end{cases}
$$

where

$$
\begin{aligned}
\lambda_{ \pm}(v) & =\frac{v}{2} \pm \frac{1}{2} \sqrt{v^{2}-4 \mu}=\frac{v}{2} \pm \frac{1}{2} \sqrt{v^{2}-\left(v^{*}\right)^{2}}, \text { for } \mu>0 \\
\lambda^{*}\left(v^{*}\right) & =\lambda_{ \pm}\left(v^{*}\right)=\frac{v^{*}}{2}
\end{aligned}
$$

The behavior of $\Phi_{0}$ for $v=v^{*}$ results from the fact that precisely at the so-called pulled velocity $v^{*}$, the two roots $\lambda_{ \pm}$coincide.

While for an arbitrary velocity $v>v^{*}$ the term $A_{1}(v)$ in $(28)$ will be nonzero, so that the asymptotic behavior of $\Phi_{0}$ is as $e^{-\lambda_{-} \xi}$, the pushed front solution — if it exists — is precisely the solution with a well-defined value $v=v^{\dagger}$ at which $A_{1}\left(v^{\dagger}\right)=0$. Note that for $\mu<0$, we have $\lambda_{-}<0$, so that 
the relevant front solution in the range $\mu<0$ has $A_{1}(v)=0$; thus the pushed front solution for $\mu>0$ is precisely the analytic continuation of this front solution to the regime $\mu>0$. If such a solution with $A_{1}\left(v^{\dagger}\right)=0$ exists, it is the dynamically selected one from steep initial conditions [31]. Moreover, these solutions decay for $\xi \gg 1$ as $e^{-\lambda_{+} \xi}$, i.e., faster than $e^{-v^{\dagger} \xi / 2}$. As a result, integrands in (27) like $e^{v^{\dagger} \xi}\left(\partial_{\xi} \Phi_{0}\right)^{2}$ or $e^{v^{\dagger} \xi}\left(\partial_{\xi} \Phi_{0}\right) g\left(\Phi_{0}, \xi\right) \propto e^{v^{\dagger} \xi}\left(\partial_{\xi} \Phi_{0}\right) \Phi_{0}$ for $\xi \rightarrow \infty$ are integrable, as

$$
e^{v^{\dagger} \xi}\left(\frac{\partial \Phi_{0}}{\partial \xi}\right)^{2} \stackrel{\xi \gg 1}{\simeq} e^{v^{\dagger} \xi} e^{-2 \lambda_{+} \xi}=e^{-\sqrt{\left(v^{\dagger}\right)^{2}-\left(v^{*}\right)^{2}}} \xi \stackrel{\xi \rightarrow \infty}{\longrightarrow} 0 .
$$

Thus, for a pushed front both criteria for a solvability analysis of a perturbation theory are satisfied: the spectrum of the stability operator is gapped and the solvability integrals converge properly.

In passing, we note that the adjoint mode $\chi$ itself does not decay to zero for large $\xi$ in the supercritical range $\mu>0$, since

$$
\chi \stackrel{\xi \gg 1}{\simeq} e^{v^{\dagger} \xi} \frac{\partial \Phi_{0}}{\partial \xi} \sim e^{\left(v^{\dagger}-\sqrt{\left(v^{\dagger}\right)^{2}-\left(v^{*}\right)^{2}}\right) \xi / 2} \stackrel{\xi \rightarrow \infty}{\longrightarrow} \infty .
$$

For our perturbation theory this is no problem as long as the inner product that defines the adjoint operator converges for $\xi \rightarrow \pm \infty$. Eq. (31) shows that this is indeed the case.

While the solvability integrals converge properly for pushed fronts, they do not for pulled fronts, as according to (30)

$$
e^{v^{*} \xi}\left(\frac{\partial \Phi_{0}}{\partial \xi}\right)^{2} \stackrel{\xi \gg 1}{\simeq} \xi^{2} e^{v^{*} \xi} e^{-2 \lambda_{*} \xi}=\xi^{2} \stackrel{\xi \rightarrow \infty}{\longrightarrow} \infty .
$$

As we already anticipated from the power law relaxation of pulled fronts, standard perturbation theory used to derive a MBA does not apply to pulled fronts.

One could, of course, regularize the solvability integrals by first introducing a cutoff $\xi_{c}$, and taking the cutoff to infinity as the end of the calculation 663. Whether such an approach yields sensible results, depends on the situation under consideration. If, e.g., this procedure is applied blindly to a solvability expression of the type (27), one finds that the changes in the nonlinear terms of the equation give no contribution - in fact, since only the divergent terms 
survive, this procedure amounts to calculating the changes in $v^{*}$ in perturbation theory for changes in the parameters in the linearized equation. Since $v^{*}$ can more easily be calculated explicitly from Eq. (1) such a calculation has no particular value.

In fact, the divergence of the solvability integrals and the absence of a characteristic time scale for the internal front dynamics are deeply related. From (25) it is easily seen that the continuous spectrum defined by $L \phi_{\sigma}=$ $-\sigma \phi_{\sigma}$, is bounded from below by $\sigma_{0}=\left(v^{2}-v^{* 2}\right) / 4$. For $\sigma(k)=\omega_{0}+k^{2}$, the eigenfunctions take the form of Fourier modes $\phi_{\sigma(k)} \propto e^{ \pm i k \xi}$ in the leading edge region $\xi \gg 1$. Hence for $v=v^{*}$, the gap $\sigma_{0}$ of the spectrum vanishes, and all the eigenfunctions of $L$ are essentially plane waves in the semi-infinite leading edge. One finds furthermore [31], that generic perturbations of pulled planar fronts $\Phi_{0}$ are even outside the Hilbert space spanned by the eigenfunctions $\phi_{\sigma}$. In this case, the long time dynamics cannot easily be understood in terms of the eigenfunctions of $L$. One rather should directly study the linearized equation

$$
\varepsilon \partial_{t} \phi=\left[\varepsilon^{2} \nabla^{2}+\left.\frac{\partial f(\phi, u)}{\partial \phi}\right|_{\phi=0}\right] \phi+O\left(\phi^{2}\right)
$$

valid in the leading edge. In this formulation, the nonlinear region of the front interior plays the role of a boundary condition for the leading edge [31, 57]. As a result one finds predictions like (1) - (4). Note finally, that the leading edge extends on the same outer length scale on which also $u$ varies. This demonstrates why it is not possible to eliminate the dynamics of a pulled front in a moving boundary approximation - independent of how thin the front is.

\section{Generalization of the solvability analysis and of its break-down}

In the previous sections, we have traced the main steps in the derivation of a MBA for two coupled equations that have been studied as phase field models for solidification. In this case, the inner equation for the order parameter reduces to the well-known nonlinear diffusion equation studied first by Fisher and Kolmogorov et al. [64, 65, 66], and the nonhermitian linear operator $L$ could be transformed to a hermitian operator $\tilde{L}$. This allowed us to obtain the 
left zero mode $\chi$ of $L$ explicitly. When one considers higher order dynamical equations or sets of coupled equations for the inner front region, it is usually not possible to find the adjoint mode explicitly. Nevertheless, we show in this section that the same conclusions hold more generally.

We consider a case where one has a vector $\vec{\phi}(x, t)$ of dynamical fields, that in the long time limit can approach a planar uniformly translating front profile $\vec{\Phi}_{0}(\xi)$ between the homogeneous stationary states $\vec{\phi}^{ \pm}=\vec{\Phi}_{0}( \pm \infty)$. The front solution $\vec{\Phi}_{0}(\xi)$ with $\xi=x-v t$ obeys a set of o.d.e.'s, and because of translation invariance $d \vec{\Phi}_{0}(\xi) / d \xi$ is again a zero mode of the linear matrix operator $\mathbf{L}$, obtained by linearizing the o.d.e.'s about the front solution $\vec{\Phi}_{0}(\xi)$ :

$$
\mathbf{L}\left(\frac{d}{d \xi}, \frac{d^{2}}{d \xi^{2}}, \frac{d^{3}}{d \xi^{3}}, \cdots ; \vec{\Phi}_{0}(\xi)\right) \cdot \frac{d \vec{\Phi}_{0}(\xi)}{d \xi}=0 .
$$

If a front $\vec{\Phi}_{0}(\xi)$ is perturbed by external forces, other coherent structures or curvature effects, one generally encounters equations like

$$
\mathbf{L} \cdot \vec{\phi}_{1}=\vec{g}_{1}
$$

in a perturbation expansion about $\vec{\Phi}_{0}$. In our example above, $\vec{g}_{1}$ decayed essentially like $\vec{\Phi}_{0}$ and $d \vec{\Phi}_{0} / d \xi$ as $\xi \rightarrow \infty$, and we only study such cases here. As is well known, such linear equations are solvable provided the right hand side is orthogonal to the kernel (null space) of $L$. The existence of a left zero mode $\vec{\chi}$ of $\mathbf{L}$ therefore generally leads to the solvability condition

$$
\int_{-\infty}^{\infty} d \xi \vec{\chi} \cdot \vec{g}_{1}=0 \quad, \quad \vec{g}_{1} \stackrel{\xi \rightarrow \infty}{\sim} \mathbf{Q} \cdot \frac{d \vec{\Phi}_{0}}{d \xi},
$$

(where the matrix $\mathbf{Q}$ contains some slowly varying fields), which relates parameters of the expansion as in (27). So we now address the question of the existence of the left zero mode $\vec{\chi}$ of $\mathbf{L}$, which is defined through $\mathbf{L}^{\dagger} \cdot \vec{\chi}=0$. In other words: $\vec{\chi}$ is the zero mode of the adjoint operator $\mathbf{L}^{\dagger}$ obtained by partial integration,

$$
\int_{-\infty}^{\infty} d \xi \vec{b} \cdot(\mathbf{L} \cdot \vec{a})=\int_{-\infty}^{\infty} d \xi\left(\mathbf{L}^{\dagger} \cdot \vec{b}\right) \cdot \vec{a}
$$

For this definition to hold, the integrals have to converge and the boundary terms that arise from performing the partial integrations all have to vanish. This imposes conditions on the allowed behavior of $\vec{b}$, given the asymptotic 
behavior of $\vec{a}$ : the product of these terms has to decay sufficiently rapidly for $\xi \rightarrow \pm \infty$.

In general, there is no particular simplifying relation between $\mathbf{L}$ and $\mathbf{L}^{\dagger}$; e.g., a term $f\left(\Phi_{0}\right) d / d \xi$ in $\mathbf{L}$ gives rise to a term $-\left(d f\left(\Phi_{0}\right) / d \xi\right)-f\left(\Phi_{0}\right) d / d \xi$ in $\mathbf{L}^{\dagger}$. As a result, there is in general no simple relation between the left and right eigenmodes. However, since $\vec{\Phi}_{0}(\xi)$ approaches the constant vectors $\vec{\phi}^{ \pm}$ for $\xi \rightarrow \pm \infty$, the operators $\mathbf{L}$ and $\mathbf{L}^{\dagger}$ asymptotically are linear operators with constant coefficients, so that

$$
\begin{gathered}
\lim _{\xi \rightarrow \pm \infty} \quad L_{i j}^{\dagger} \quad\left(\frac{d}{d \xi}, \frac{d^{2}}{d \xi^{2}}, \frac{d^{3}}{d \xi^{3}}, \cdots ; \vec{\phi}_{v}^{0}(\xi)\right)=L_{i j}^{\dagger}\left(\frac{d}{d \xi}, \frac{d^{2}}{d \xi^{2}}, \frac{d^{3}}{d \xi^{3}}, \cdots ; \phi^{ \pm}\right)= \\
=L_{j i}\left(-\frac{d}{d \xi}, \frac{d^{2}}{d \xi^{2}},-\frac{d^{3}}{d \xi^{3}}, \cdots ; \phi^{ \pm}\right) .
\end{gathered}
$$

Moreover, in this limit, the operator $\mathbf{L}$ is exactly the same as the one that one obtains from linearizing the set of o.d.e.'s for $\vec{\Phi}_{0}$ around the homogeneous stationary states $\vec{\phi}^{ \pm}$. Therefore both $\vec{\Phi}_{0}$ and the right zero mode $d \vec{\Phi}_{0} / d \xi$ of $\mathbf{L}$ are asymptotically for $\xi \rightarrow \pm \infty$ just sums of simple exponentials of the form

$$
d \vec{\Phi}_{0} / d \xi \quad \stackrel{\xi \rightarrow \pm \infty}{\simeq} \sum_{n=1}^{N} \vec{a}_{n}^{ \pm} e^{-\lambda_{n}^{ \pm} \xi},
$$

where the eigenvalues $\lambda_{n}^{ \pm}$are determined by the characteristic polynomial of degree $N$

$$
\operatorname{det} \mathbf{L}\left(-\lambda_{n}^{ \pm}, \lambda_{n}^{ \pm 2},-\lambda_{n}^{ \pm 3}, \cdots ; \vec{\phi}^{ \pm}\right)=0
$$

The asymptotic behavior of an adjoint zero mode $\vec{\chi}$ follows immediately from the symmetry relation (39). If we write the asymptotics for $\xi \rightarrow \pm \infty$ of $\vec{\chi}$ as

$$
\vec{\chi} \stackrel{\xi \rightarrow \pm \infty}{\simeq} \quad \sum_{n=1}^{N} \vec{b}_{n}^{ \pm} e^{-\bar{\lambda}_{n}^{ \pm} \xi}
$$

then the eigenvalues $\bar{\lambda}_{n}^{ \pm}$are determined by the eigenvalue equation

$$
\operatorname{det} \mathbf{L}^{\dagger}\left(-\bar{\lambda}_{n}^{ \pm}, \bar{\lambda}_{n}^{ \pm 2},-\bar{\lambda}_{n}^{ \pm 3}, \cdots ; \vec{\phi}^{ \pm}\right)=0
$$

which in view of (39) and the fact that $\operatorname{det} \mathbf{L}=\operatorname{det} \mathbf{L}^{\dagger}$ immediately yields

$$
\bar{\lambda}_{n}^{ \pm}=-\lambda_{n}^{ \pm}
$$


Let us now investigate the asymptotic behavior of products $\vec{b} \cdot \vec{a}$ of left modes $\vec{b}$ and right modes $\vec{a}$, which is required for the existence and definition of the adjoint operator and modes. Assume that the eigenvalues are ordered as $\operatorname{Re} \lambda_{n+1}^{ \pm} \geq \operatorname{Re} \lambda_{n}^{ \pm}$. A pushed or bistable front is a discrete solution with asymptotic behavior

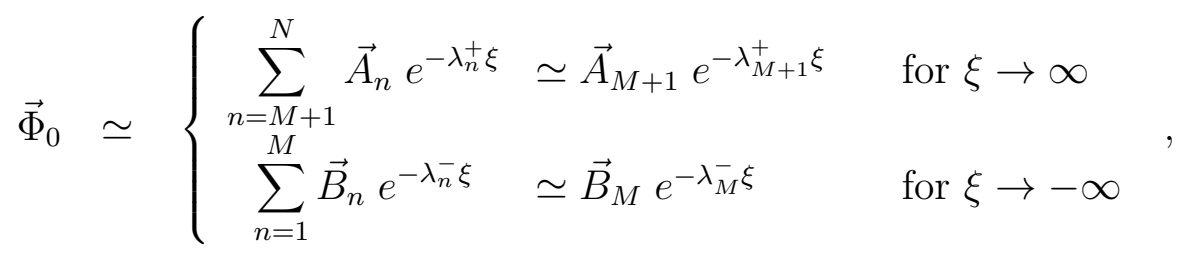

that can be constructed from (40) for a particular value of $v=v^{\dagger}$. The right zero mode $d \vec{\Phi}_{0} / d \xi$ obviously has the same asymptotic behavior. In this expression, the eigenvalues $\lambda_{1}^{-}, \cdots, \lambda_{M}^{-}$for $\xi \rightarrow \infty$ are all the eigenvalues with negative real part so that the exponentials converge, while on the right for $\xi \rightarrow \infty$ all $\lambda_{M+1}^{+}, \cdots, \lambda_{N}^{+}$have positive real parts. The existence of $M$ modes on the left and $N-M+1$ modes on the right is a reflection of the fact that the bistable or pushed front solution is an isolated (discrete) solution [31].

At this point, there is only one difference between bistable fronts and pushed fronts propagating into an unstable state: for the former, $\operatorname{Re} \lambda_{M}^{+}<0$ so that this mode is not present because it corresponds to a diverging behavior, while for a pushed front propagating into an unstable state, $\operatorname{Re} \lambda_{M}^{+}>0$ but $A_{M}^{+}=0$ by definition [31].

A product of this right mode with a left mode converges to zero at $\pm \infty$, if the left zero mode behaves asymptotically like

$$
\vec{\chi} \simeq\left\{\begin{array}{lll}
\sum_{n=1}^{M} \vec{C}_{n} e^{\lambda_{n}^{+} \xi} & \simeq \vec{C}_{M} e^{\lambda_{M}^{+} \xi} & \text { for } \xi \rightarrow \infty \\
\sum_{n=M+1}^{N} \vec{D}_{n} e^{\lambda_{n}^{-} \xi} & \simeq \vec{D}_{M+1} e^{\lambda_{M+1}^{-} \xi} & \text { for } \xi \rightarrow-\infty
\end{array}\right.
$$

One easily verifies by counting the dimensions of stable and unstable manifolds in the two asymptotic regions, that also $\chi$ belongs to a discrete spectrum, independent of the value of $M$, and that in general the divergent term $\sim e^{\lambda_{M}^{+} \xi}$ is needed for this mode to exist. Indeed, the textbook argument

$$
\begin{gathered}
\mathbf{L} \cdot \vec{\phi}_{m}=\sigma_{m} \vec{\phi}_{m} \quad, \quad \mathbf{L}^{\dagger} \cdot \vec{\chi}_{l}=\sigma_{l}^{\dagger} \vec{\chi}_{l}, \\
\sigma_{l}^{\dagger} \int \vec{\chi}_{l} \cdot \vec{\phi}_{m}=\int\left(\mathbf{L}^{\dagger} \cdot \vec{\chi}_{l}\right) \cdot \vec{\phi}_{m}=\int \vec{\chi}_{l} \cdot\left(\mathbf{L} \cdot \vec{\phi}_{m}\right)=\sigma_{m} \int \vec{\chi}_{l} \cdot \vec{\phi}_{m}
\end{gathered}
$$


shows that the eigenvalues $\sigma_{l}^{\dagger}=\sigma_{m}$ equal each other, if the product of the eigenfunctions $\int \vec{\chi}_{l} \cdot \vec{\phi}_{m}$ is finite and likewise that eigenfunctions with different eigenvalues are orthogonal. Application of simple "counting arguments" 556, 31] for the existence and multiplicity of solutions of o.d.e.'s shows that (47) implies that associated with the discrete right zero mode of a pushed or bistable front solution, there is in general an isolated (discrete) left zero mode of $\mathbf{L}^{\dagger}$ with a nonzero divergent term (47).

This reasoning does not work for a pulled front, where the zero mode of $\mathbf{L}$ is part of a continuous spectrum with the same asymptotic decay properties at $\xi \rightarrow \pm \infty$. The same counting argument as above now yields, that in general no left zero mode of $\mathbf{L}^{\dagger}$ exists.

This formal argument is supported by the observation, that a solvability integral for a pushed front diverges as the pushed velocity $v^{\dagger}$ approaches the pulled velocity $v^{*}$ (1): Generally, the velocity $v$ will appear as a parameter in the characteristic polynomial (41). If we consider the $\lambda_{n}=\lambda_{n}^{+}$as functions of $v$, then according to the general scenario of front propagation into unstable states 31 the pulled velocity is associated with a minimum of the curve $v\left(\lambda_{M}\right)$ where $\lambda_{M}$ is the root of (41) with the smallest positive real part. Hence for $v \gtrsim v^{*}$ and uniformly translating fronts with $\lambda_{M}$ and $\lambda_{M+1}$ real, we have

$$
\begin{aligned}
\lambda_{M}(v) & =\lambda^{*}-\frac{2}{v^{\prime \prime}} \sqrt{v-v^{*}}+\cdots, \\
\lambda_{M+1}(v) & =\lambda^{*}+\frac{2}{v^{\prime \prime}} \sqrt{v-v^{*}}+\ldots,
\end{aligned}
$$

where

$$
v^{\prime \prime}=\left.\frac{d^{2} v\left(\lambda_{M}\right)}{d \lambda_{M}^{2}}\right|_{\lambda^{*}}
$$

is the curvature of $v\left(\lambda_{M}\right)$ in the minimum that determines $v^{*}$ and $\lambda^{*}$ (see [31], section V.C.2). It hence is a positive constant.

In complete analogy with our earlier discussion in section 3 , the general scenario for front propagation into unstable states is that while the asymptotic decay for $\xi \gg 1$ is as $e^{-\lambda_{M} \xi}$ for an arbirary velocity $v$, a pushed front solution exists if for some velocity $v^{\dagger}>v^{*}$, there is a front solution whose asymptotic large $\xi$ behavior is as $e^{-\lambda_{M+1} \xi}$ in agreement with (45).

If there is no such pushed front solution, then starting from "steep" initial conditions the selected front velocity is $v^{*}$; the asymptotic front profile with 
this velocity is then

$$
\vec{\Phi}_{0}(\xi) \stackrel{\xi \gg 1}{\simeq}(\vec{\alpha} \xi+\vec{\beta}) e^{-\lambda_{M} \xi}, \lambda_{M}=\lambda_{M+1},
$$

in analogy with (28).

As we discussed above, for a pushed front, there is in general a discrete left zero mode (46) with asymptotic behavior $\chi \sim e^{\lambda_{M} \xi}$ for large $\xi$. In spite of this divergence, the product of left and right modes converges as

$$
\begin{aligned}
& \vec{\chi} \cdot \mathbf{Q} \cdot \frac{d \vec{\phi}_{0}}{d \xi} \quad \stackrel{\xi \gg 1}{\simeq} \quad e^{\left(\lambda_{M}-\lambda_{M+1}\right) \xi} \vec{C}_{M} \cdot \mathbf{Q} \cdot \vec{A}_{M+1} \\
& \sim e^{-\left(4 / v^{\prime \prime}\right) \sqrt{v^{\dagger}-v^{*}}} \xi \stackrel{\xi \rightarrow \infty}{\longrightarrow} 0 \quad\left(v \gtrsim v^{*}\right),
\end{aligned}
$$

and solvability conditions generally can be derived.

Just as we saw in the previous sections, the present analysis also shows that as $v^{\dagger}$ approaches $v^{*}$ from above, the solvability integrals converge less and less fast until, at $v^{*}$, we have according to (52)

$$
\vec{\chi} \cdot \mathbf{Q} \cdot \frac{d \vec{\Phi}_{0}}{d \xi} \stackrel{\xi \gg 1}{\sim} \quad \xi^{2} \stackrel{\xi \rightarrow \infty}{\longrightarrow} \infty .
$$

in complete analogy with our earlier result (33) for the example discussed in section 3 .

\section{Conclusions and outlook}

In contrast to "bistable" or pushed fronts, the dynamics of pulled fronts is determined essentially in the leading edge. This was recently shown to imply a general power law relaxation of pulled fronts. In this paper, we have shown that this in turn entails that pulled fronts lack the separation of time scales necessary for the applicability of the usual MBA, and that solvability integrals diverge when a front is pulled.

It is important to stress that one should not simply view this negative result as a formal problem - rather, one should take this conclusion as a signal that the pattern dynamics involving the motion of pulled fronts poses interesting new physical questions with possibly surprising non-standard answers. 
As a first simple illustration of this, consider the uncoupled F-KPP equation (7) in two dimensions with $\varepsilon=1$ and $f=\phi-\phi^{3}$. If one starts with a radially symmetric steep initial condition, e.g., $\phi(r, t=0)=\exp \left(-r^{2}\right)$, then this front will spread out in a circularly symmetric way. According to (27) the curvature correction will then give a contribution $-1 / r=-1 /\left(v^{*} t\right)=-1 /(2 t)$ to the velocity at large times. However, in addition to that, there is a contribution $-3 /(2 t)$ of the same order of magnitude from the power law relaxation (3) , as $\lambda^{*}=1$ in this case. Thus, due to the combination of the power law relaxation and the curvature correction, the front velocity $v^{*}$ will be approached asymptotically as $v(t)=v^{*}-2 / t$ 67]!

In the example above of a circularly symmetric pattern without any coupling to other fields, the relaxation and curvature effects can be simply added up, but for a less trivial patterns whose shape is changing in time, the proper description is far from obvious. Nontrivial patterns where pulled front propagation plays a dominant role occur e.g. in streamer discharges 48]. Hence new analytical tools have to be developed for a moving boundary like description of these finger-like patterns. Work in progress [68] suggests that the limit of zero electron diffusion creating shock-like electron fronts is a valuable approximation for negatively charged streamers.

A recent illustration of the fact that the nonexistence of solvability integrals signals a transition to qualitatively different dynamical behavior is given by the behavior of fronts in the presence of multiplicative noise [29, 30]. Pushed fronts in the presence of multiplicative noise show regular diffusive behavior due to the noise being summed over the finite interior front region, and their diffusion coefficient can be expressed in terms of solvability type integrals 29. In contrast, fully relaxed pulled fronts in an infinite system do not diffuse at all, and if a front with pulled dynamics starts from a local (or "sufficiently steep" [31]) initial condition, it is subdiffusive [30]: the root mean square displacement of pulled fronts increases with time as $t^{1 / 4}$, not as $t^{1 / 2}$. This prediction was first suggested by using a time-dependent cutoff $\xi_{c}(t) \sim \sqrt{t}$ in the solvability expression for the diffusion coefficient that is valid for pushed fronts. The motivation for this time-dependent cutoff comes from the relaxation analysis of pulled fronts given in [31]. Hence, this example illustrates both that using a cutoff in the solvability integrals sometimes can yield sensible results, and that the behavior of pulled fronts can be qualitatively different from those of pushed fronts.

We finally note that these considerations also have implications for numer- 
ical codes. In cases where a MBA applies in the limit in which the front width is taken to zero, numerical codes with adaptive gridsize refinement in the interior front region, where gradients are large, are quite efficient. For pulled fronts, however, solutions with a too coarse basic grid give inaccurate front velocities. For these, the refinement has to be done ahead of the front, in the leading edge [69]!

\section{Acknowledgement}

The work of UE was supported by the Dutch research foundation NWO and by the EU-TMR network "Patterns, Noise and Chaos".

\section{References}

[1] P. C. Fife, Dynamics of Internal Layers and Diffusive Interfaces (SIAM, Philadelphia, 1988)

[2] A. Karma and W.-J. Rappel, Phys. Rev. E 53, R3017 (1996); Phys. Rev. E 57, 4323 (1998).

[3] J. D. Buckmaster and G. S. S. Lundford, Theory of Laminar Flames (Cambridge University Press, Cambridge, 1982).

[4] R. Kobayashi, Physica 63D, 410 (1993).

[5] J. Müller and M. Grant, Phys. Rev. Lett. 82, 1736 (1999).

[6] K. Kassner and C. Misbah, Europhys. Lett. 46, 217 (1999).

[7] R. González-Cinca, L. Rámirez-Piscina, J. Casademunt, A. HernándezMachado, L. Kramer, T. Tóth-Katona, T. Börzsönyi, and Á Buka, Physica D 99359 (1996).

[8] P. W. Bates, P. C. Fife, and C. K. R. T. Jones, Physica D 104, 1 (1997).

[9] J. D. Gunton, M. San Miguel and P. S. Sahni, in: Phase transitions and critical phenomena, vol. 8, C. Domb and J. L. Lebowitz, eds. (Academic, New York, 1983). 
[10] A. J. Bray, Adv. Phys. 43, 357 (1994).

[11] J. S. Langer, in: Chance and Matter, ed. J. Souletie (North-Holland, Amsterdam, 1987); J. S. Langer, in: Solids far from equilibrium, C. Godreche, ed. (Cambridge University Press, Cambridge, 1992).

[12] P. Pelcé, Dynamics of curved fronts (Academic, Boston, 1988).

[13] D. A. Kessler, J. Koplik and H. Levine, Adv. Phys. 37, 255 (1988).

[14] Y. Pomeau and M. Ben Amar, Dendritic growth and related topics, in: Solids far from Equilibrium, C. Godreche, Ed. (Cambridge University Press, Cambridge, 1992).

[15] E. A. Brener and V. I. Mel'nikov, Adv. Phys. 40, 53 (1991).

[16] B. Caroli, C. Caroli and B. Roulet, Instabilities of planar solidification fronts, in: Solids far from Equilibrium, ed. G. Godrèche (Cambridge University Press, Cambridge, 1992)

[17] K. Kassner, Pattern Formation in Diffusion-Limited Crystal Growth (World Scientific, Singapore, 1996).

[18] D. Kandel and J. D. Weeks, Phys. Rev. Lett. 721678 (1994); 743632 (1995).

[19] C. Misbah and A. Valance, Phys. Rev. E 49, 166 (1972).

[20] C. Misbah, O. Pierre-Louis, and A. Pimpinelli, Phys. Rev. B 51, 17283 (1995).

[21] G. Zocchi, P. Tabeling and M. Ben Amar, Phys. Rev. Lett. 69, 601 (1992).

[22] M. Ben Amar, Phys. Fluids A 42641 (1992).

[23] E. Meron, Phys. Rep. 218, 1 (1992).

[24] O. Törnkvist and E. Schröder, Phys. Rev. Lett. 78, 1908 (1997).

[25] I. S. Aranson, H. Chaté, L.-H. Tang, Phys. Rev. Lett. 80, 2646 (1998).

[26] A. Malevanets, A. Careta, and R. Kapral, Phys. Rev. E 52, 4724 (1995). 
[27] Á. Tóth, D. Horváth, and W. van Saarloos, Lateral instabilities of cubic autocatalytic reaction fronts in constant electric field, to appear in $\mathrm{J}$. Chem. Phys.

[28] I. V. Biktasheva, Yu. E. Elkin and V. N. Betashev, Phys. Rev. E. 57, 2656 (1998).

[29] J. Armero, J. Casademunt, L. Ramírez-Picina, and J. M. Sancho, Phys. Rev. E. 58, 5494 (1998).

[30] A. Rocco, U. Ebert, and W. van Saarloos (unpublished).

[31] U. Ebert and W. van Saarloos, Phys. Rev. Lett. 80, 1650 (1998); Front propagation into unstable states: Universal algebraic convergence towards uniformly translating pulled fronts [preprint submitted to Physica D].

[32] C. M. Bender and S. A. Orszag, Advanced Mathematical Methods for Scientists and Engineers (McGraw-Hill, New York, 1978).

[33] M. C. Cross and P. C. Hohenberg, Rev. Mod. Phys. 65, 851 (1992).

[34] A. C. Newell, T. Passot and J. Lega, Ann. Rev. Fluid Mech. 25399 (1993).

[35] W. van Saarloos, Phys. Rep. 301, 9 (1998).

[36] A. N. Bers, in: Handbook of Plasma Physics, M. N. Rosenbluth and R. Z. Sagdeev, eds. (North-Holland, Amsterdam, 1983).

[37] E. M. Lifshitz and L.P. Pitaevskii, Physical Kinetics (Pergamon, New York, 1981).

[38] A. N. Stokes, Math. Biosci. 31, 307 (1981).

[39] E. Ben-Jacob, H.R. Brand, G. Dee, L. Kramer, and J.S. Langer, Physica D 14, 348 (1985).

[40] W. van Saarloos, Phys. Rev. A 39, 6367 (1989).

[41] G. C. Paquette, L.-Y. Chen, N. Goldenfeld and Y. Oono, Phys. Rev. Lett. 72, 76 (1994). 
[42] T. R. Powers and R. E. Goldstein, Phys. Rev. Lett. 78, 2555 (1997).

[43] G. Ahlers and D. S. Cannell, Phys. Rev. Lett. 50, 1583 (1983); M. Niklas, M. Lücke, and H. Müller-Krumbhaar, Phys. Rev. A 40, 493 (1989).

[44] J. Fineberg and V. Steinberg, Phys. Rev. Lett. 58, 1332 (1987).

[45] C. Mathis, M. Provansal, and L. Boyer, J. Phys. Lett. 45 L483 (1984); G. S. Triantafyllou, K. Kupfer, and A. Bers, Phys. Rev. Lett. 59, 1914 (1987); T. Leweke, M. Provansal, G. D. Miller, and C. H. K. Williamson, Phys. Rev. Lett. 78, 1259 (1997).

[46] A. Couairon and J. M. Chomaz, Physica D 108, 236 (1997); Phys. Rev. Lett. 79, 2666 (1997).

[47] J. E. Maclennan, N. A. Clark, and T. Carlsson, in: Solitons in Liquid crystals, L. Lam, ed. (Springer, Berlin, 1991); P. Cladis and W. van Saarloos, ibidem; W. van Saarloos, M. van Hecke and R. Hołyst, Phys. Rev. E 52, 1773 (1995).

[48] U. Ebert, W. van Saarloos and C. Caroli, Phys. Rev. Lett. 77, 4178 (1996); Phys. Rev. E 55, 1530 (1997).

[49] Y. Tu and M. C. Cross, Phys. Rev. Lett. 69, 2515 (1992).

[50] E. K. H. Salje, J. Phys.: Condens. Matter 5, 4775 (1993).

[51] R. C. Ball and R. L. H. Essery, J. Phys.: Condens. Matter 2, 10303 (1990); R. A. L. Jones, L. J. Norton, E. J. Kramer, F. S. Bates and P. Wiltzius, Phys. Rev. Lett. 66, 1326 (1991).

[52] S. J. Di Bartolo and A. T. Dorsey, Phys. Rev. Lett. 77, 4442 (1996).

[53] A. Torcini, P. Grassberger, and A. Politi, J. Phys. A 27, 4533 (1995).

[54] J. Krug and P. Meakin, Phys. Rev. A 43, 900 (1991).

[55] K. Nozaki and N. Bekki, Phys. Rev. Lett. 51, 271 (1983).

[56] W. van Saarloos and P. C. Hohenberg, Physica D 56, 303 (1992). 
[57] C. Storm, W. Spruijt, U. Ebert and W. van Saarloos, [submitted to Phys. Rev. Lett.].

[58] D. Carpentier and P. Le Doussal, Phys. Rev. Lett. 81, 2558 (1998).

[59] R. van Zon, H. van Beijeren, and Ch. Dellago, Phys. Rev. Lett. 80, 2035 (1998).

[60] S. M. Allen and J. W. Cahn, Acta Metall. 27, 1085 (1979).

[61] R. Kupferman, O. Shochet, E. Ben-Jacob, and A. Schuss, Phys. Rev. B 46, 16045 (1992).

[62] Kupferman et al. [61] use the same $\varepsilon$ scaling in (7), but even allow for $\kappa=O\left(\varepsilon^{-1}\right)$. However, this is not consistent with the standard choice of the coordinate system on the inner scale [1] as also used by them and by us below.

[63] L.-Y. Chen, N. Goldenfeld, Y. Oono, and G. Paquette, Physica A 204, 111 (1994).

[64] R. A. Fisher, Ann. Eugenics 7, 355 (1937).

[65] A. Kolmogorov, I. Petrovsky, and N. Piscounoff, Bulletin de l'université d'état à Moscou, Ser. int., Section A, Vol. 1 (1937).

[66] D. G. Aronson and H. F. Weinberger, Adv. Math. 30, 33 (1937).

[67] This prediction was realized independently and tested numerically by B. Derrida (private communication).

[68] M. Arrayás and U. Ebert (unpublished).

[69] W. Hundsdorfer and U. Ebert (unpublished). 